\title{
DIOPHANTINE APPROXIMATION WITH PARTIAL SUMS OF POWER SERIES
}

\author{
BRUCE C. BERNDT, SUN KIM, M.TIP PHAOVIBUL, AND ALEXANDRU ZAHARESCU
}

\section{Introduction AND StATEMENTS OF RESUlts}

Consider the exponential generating function of a sequence $\left\{a_{n}\right\}, n \geq 0$,

$$
G(s):=\sum_{n=0}^{\infty} \frac{a_{n}}{n !} s^{n}, \quad s \in \mathbb{C} .
$$

For each nonzero rational number $r$, in [1], we considered the problem of approximating $G(r)$ with partial sums of the series (1.1). In the case that $a_{n} \equiv 1$ and $s=1$, we asked how well one can approximate $e$ by the partial sums $\sum_{\ell=0}^{n} \frac{1}{\ell !}$. J. Sondow [6] conjectured that exactly two of these partial sums are also convergents to the continued fraction of $e$. Among several results, Sondow and K. Schalm [7], proved that for almost all positive integers $n$, the partial sum $\sum_{\ell=0}^{n} \frac{1}{\ell !}$ is not a convergent to the continued fraction of $e$. Thus, the probability of obtaining a convergent to the continued fraction of $e$ upon randomly choosing one of the first $n$ partial sums of the power series of $e$ tends to zero as $n \rightarrow \infty$. Knowledge of the continued fraction of $e^{2 / a}$, where $a$ is a nonzero integer, and the best possible diophantine approximation of $e^{2 / a}$, discovered by S. Ramanujan [5] and rediscovered by C. S. Davis [4], enabled the authors to prove in [2] that at most $O_{a}(\log M)$ of the first $M$ convergents to the continued fraction of $e^{2 / a}$ are also partial sums of the corresponding power series. In [1], we considered general hypergeometric functions ${ }_{p} F_{p}\left(a_{1}, \ldots, a_{p} ; b_{1}, \ldots, b_{p} ; r\right)$ and showed that among their first $N$ partial sums, no more than $O(\log N)$ are convergents to the continued fraction of ${ }_{p} F_{p}\left(a_{1}, \ldots, a_{p} ; b_{1}, \ldots, b_{p} ; r\right)$. Observe that this result includes $e^{2 / a}$ as a special case and that this particular corollary is a dual of the aforementioned result established in [2]. Moreover, when $a_{n}$ is a real Dirichlet character or when $\left\{a_{n}\right\}, n>0$, are the coefficients of an $L$-series attached to an elliptic curve without complex multiplication, we proved similar theorems in [1]. Lastly, we remark that in [2], we, in fact, proved Sondow's conjecture.

At the focal point of our study in [1] and in this paper are the following two definitions. For any rational number $\mu=a / b$, with $(a, b)=1$, consider the height $H(\mu)$ of $\mu$, given by $H(\mu)=\max \{|a|,|b|\}$. For any real number $\alpha$, and any positive real number $\delta$, denote

$$
A_{\alpha, \delta}:=\left\{\mu \in \mathbb{Q}:|\alpha-\mu|<\frac{1}{H(\mu)^{1+\delta}}\right\} .
$$

In the present paper, we greatly expand our outlook in two different directions. First, we complement the results from [1] with a positivity argument. For sequences $\left\{a_{n}\right\}_{n \in \mathbb{N}}$ of nonnegative integers, we first establish a general result with the accuracy of the results described in our opening paragraph. For each such sequence, we define the gap function

The first author's research was partially supported by NSA grant H98230-11-1-0200.

The fourth author's research was partially supported by NSF grant DMS-0901621. 
$i(n)$ to be the number of consecutive elements equal to zero immediately following $a_{n}$, that is,

$$
i(n):=\min \left\{m: m \geq n+1, a_{m} \neq 0\right\}-n-1 .
$$

Thus, for sequences in which $a_{n}>0, n \geq 1$, the gap function is identically equal to zero. In the sequel, we consider sequences for which the gap function satisfies

$$
\lim _{n \rightarrow \infty} \frac{i(n)}{n}=0 .
$$

We shall allow the size of $a_{n}$ to be quite large. More precisely, we require that $a_{n}=O_{\epsilon}\left(n^{\epsilon n}\right)$, for any $\epsilon>0$, which we write in the form

$$
\lim _{n \rightarrow \infty} \frac{\log \left(1+a_{n}\right)}{n \log n}=0 .
$$

We then have the following result.

Theorem 1.1. Let $\left\{a_{n}\right\}, n \in \mathbb{N}$, be a sequence of nonnegative integers satisfying (1.4) and (1.5), and consider the entire function

$$
G(s):=\sum_{n=1}^{\infty} \frac{a_{n}}{n !} s^{n} .
$$

Then for any positive rational number $r$ and any real number $\delta>0$, there is a constant $C_{1}>0$, depending only on $\delta$, and a constant $C_{2}>0$, depending on $r, \delta$, and the given sequence $\left\{a_{n}\right\}, n \in \mathbb{N}$, such that for all positive integers $N$,

$$
\#\left\{n \leq N: a_{n} \neq 0 \text { and } \sum_{\ell=1}^{n} \frac{a_{\ell}}{\ell !} r^{\ell} \in A_{G(r), \delta}\right\} \leq C_{1} \log N+C_{2} .
$$

Theorem 1.1 is applicable to a large variety of sequences of interest in number theory. We offer a few classical examples obtained from the Riemann zeta function $\zeta(s)$ and $L$ functions: $\zeta^{k}(s)=\sum_{n=1}^{\infty} d_{k}(n) n^{-s}$, where $d_{k}(n)$ denotes the number of ways of writing $n$ as a product of $k$ positive integers;

$$
\frac{\zeta(s-1)}{\zeta(s)}=\sum_{n=1}^{\infty} \frac{\varphi(n)}{n^{s}}
$$

where $\varphi(n)$ denotes Euler's $\varphi$-function; and, for any two quadratic Dirichlet characters $\chi_{1}$ and $\chi_{2}, \zeta(s) L\left(s, \chi_{1}\right) L\left(s, \chi_{2}\right) L\left(s, \chi_{1} \chi_{2}\right)$, a product which plays a key role in Siegel's theorem on possible exceptional (Siegel) zeros; see Davenport's text [3, Chapter 21].

We performed substantial calculations in support of our conjecture that, in each case, values of partial sums coincide with partial quotients of the continued fraction only a finite number of times. In particular, for integral powers of $\zeta(s)$ up to the 20th, we calculated the first 2000 partial sums and partial quotients. The table below provides a list of coalescing partial sums and partial quotients of continued fractions that we found in our calculations for powers of $\zeta(s)$. We conjecture that for any positive integral power of $\zeta(s)$, there are only a finite number of values assumed by both partial sums and partial quotients. Moreover, in each of the cases below, we conjecture that we have found all possible matchings. Observe 
that for powers $k>6$, only when $k=15$ do we find matching partial sums and partial quotients. Is it true that for $k>15$, there are no further common values?

\begin{tabular}{|c|c||c|c|}
\hline$k$ & Common Values & $k$ & Common Values \\
\cline { 3 - 4 } 1 & $\left\{1, \frac{5}{3}\right\}$ & 11 & \{\} \\
2 & $\{2\}$ & 12 & \{\} \\
3 & $\left\{3, \frac{1243}{378}\right\}$ & 13 & \{\} \\
4 & \{\} & 14 & \{\} \\
5 & $\{5\}$ & 15 & $\left\{16, \frac{27649}{1680}\right\}$ \\
6 & $\left\{5, \frac{27545}{4608}\right\}$ & 16 & \{\} \\
17 & \{\} \\
7 & \{\} & 17 & \{\} \\
8 & \{\} & 18 & \{\} \\
9 & \{\} & 19 & \{\} \\
10 & \{\} & \\
\hline
\end{tabular}

Next, for a real Dirichlet character $\chi$, we consider

$$
G(s)=G_{\chi}(s):=\sum_{n=1}^{\infty} \frac{\chi(n)}{n !} s^{n} .
$$

In [1], we conjectured that for any real Dirichlet character $\chi$ and any nonzero rational number $r$, there exist only finitely many positive integers $n$ for which the partial sum $\sum_{n=1}^{m} \frac{\chi(n)}{n !} r^{n}$ is a convergent to the continued fraction of $G_{\chi}(s)$. In connection with this conjecture, we showed in [1] that at most $O_{\chi, r}(\log N)$ of the first $N$ partial sums are also convergents to the continued fraction of $G_{\chi}(r)$. We also raised the natural problem to estimate, for large $x$, the largest number of the common values of the partial sums $\sum_{n=1}^{m} \frac{\chi(n)}{n !} r^{n}$ and the convergents to the continued fraction of $G_{\chi}(r)$ as $\chi$ runs over all real characters of conductor $\leq x$. In relation to this, we prove the following result.

Theorem 1.2. For a real Dirichlet character $\chi$, let $\ell(\chi)$ be the number of values to simultaneously be partial sums of the power series and convergents to the continued fraction of $G_{\chi}(s)$. Then,

$$
\max _{q \leq x} \max _{\chi \bmod q} \ell(\chi)>>\log \log x .
$$

In another direction, instead of studying the approximation of one particular real value of the exponential function, i.e., $e^{2 / a}$, by rational numbers, we examine the more general and delicate problem of approximating complex numbers of the form $\alpha_{1} e^{\beta_{1}}+\cdots+\alpha_{r} e^{\beta_{r}}$, where $\alpha_{1}, \ldots, a_{r}, \beta_{1}, \ldots, \beta_{r}$ are in $\overline{\mathbb{Q}}$, the algebraic closure of $\mathbb{Q}$ in $\mathbb{C}$, by partial sums of their power series. Before commencing this study, we need to describe the setting and fix notation.

For any finite field extension $K$ of $\mathbb{Q}$ contained in $\overline{\mathbb{Q}}$, we denote by $\mathcal{O}_{K}$ the ring of integers of $K$. For any ideal $J$ we let $\|J\|$ denote its norm. In what follows, we use the following notion of height of an algebraic number. Given $\theta \in \overline{\mathbb{Q}}$, there exists a unique polynomial 
$P(X)=C_{0} X^{d}+C_{1} X^{d-1}+\cdots+C_{d} \in \mathbb{Z}[X]$, irreducible over $\mathbb{Q}$, with $C_{0}>0$ and $\operatorname{gcd}\left(C_{0}, C_{1}, \ldots, C_{d}\right)=1$ such that $P(\theta)=0$. We let

$$
H(\theta):=\max \left\{C_{0},\left|C_{1}\right|, \ldots,\left|C_{d}\right|\right\} .
$$

In particular, if $\theta=a / q$ is rational, with $a, q \in \mathbb{Z}, q>0,(a, q)=1$, then $P(X)=q X-a$ and $H(\theta):=\max \{q,|a|\}$. We will also use the following terminology. Given a vector $\bar{\beta}=\left(\beta_{1}, \ldots, \beta_{r}\right)$ with $\beta_{1}, \ldots, \beta_{r} \in \overline{\mathbb{Q}}$, we will say that $\bar{\beta}$ is admissible if the numbers $\beta_{1}, \ldots, \beta_{r}$ are nonzero and distinct, and if two components $\beta_{i}$ and $\beta_{j}$ have the same largest absolute value, then the ratio $\beta_{i} / \beta_{j}$ is a root of unity.

Now let $\bar{\alpha}=\left(\alpha_{1}, \ldots, \alpha_{r}\right), \bar{\beta}=\left(\beta_{1}, \ldots, \beta_{r}\right)$, with $\alpha_{1}, \ldots, \alpha_{r}, \beta_{1}, \cdots, \beta_{r} \in \overline{\mathbb{Q}}-\{0\}$ and $\bar{\beta}$ admissible, and consider the complex number

$$
\rho=\alpha_{1} e^{\beta_{1}}+\cdots+\alpha_{r} e^{\beta_{r}} .
$$

We know from the Lindemann-Weierstrass theorem that $\rho$ is transcendental over $\mathbb{Q}$. We write $\rho$ in the form

$$
\rho=\sum_{\ell=0}^{\infty} \frac{a_{\ell}}{\ell !}
$$

where for any $\ell$,

$$
a_{\ell}=\sum_{j=1}^{r} \alpha_{j} \beta_{j}^{\ell}
$$

and consider approximations of $\rho$ by the partial sums $\sum_{\ell=0}^{n} \frac{a_{\ell}}{\ell !}$. To assess the quality of such an approximation, we consider the height $H\left(\sum_{\ell=0}^{n} \frac{a_{\ell}}{\ell !}\right)$ and the distance from $\sum_{\ell=0}^{n} \frac{a_{\ell}}{\ell !}$ to $\rho$.

For any complex number $z$ and any positive real number $\tau$, we consider the set

$$
A_{z, \tau}=\left\{\theta \in \overline{\mathbb{Q}}:|z-\theta| \leq \frac{1}{H(\theta)^{\tau}}\right\} .
$$

Note that by (1.10) and (1.11),

$$
\left|\rho-\sum_{\ell=0}^{n} \frac{a_{\ell}}{\ell !}\right| \leq \frac{1}{(n !)^{1-\epsilon}},
$$

for any fixed $\epsilon>0$ and $n$ large enough in terms of $\bar{\alpha}, \bar{\beta}$, and $\epsilon$. Also, at least in the case when $\bar{\alpha}$ and $\bar{\beta}$ have rational components,

$$
H\left(\sum_{\ell=0}^{n} \frac{a_{\ell}}{\ell !}\right) \leq(n !)^{1+\epsilon},
$$

for any fixed $\epsilon>0$ and $n$ sufficiently large in terms of $\bar{\alpha}, \bar{\beta}$, and $\epsilon$. By (1.13) and (1.14), it follows that if $\bar{\alpha}$ and $\bar{\beta}$ have rational components, then each of the sets $A_{\rho, \tau}, 0<\tau<1$, contains all but finitely many partial sums $\sum_{\ell=0}^{n} \frac{a_{\ell}}{\ell !}$. In the following, we focus on the case when $\tau>1$. Our main theorem below shows that in this case very few partial sums $\sum_{\ell=0}^{n} \frac{a_{\ell}}{\ell !}$ belong to $A_{\rho, \tau}$. More precisely, we prove the following result. 
Theorem 1.3. Let $r$ be a positive integer, let $\bar{\alpha}=\left(\alpha_{1}, \ldots, \alpha_{r}\right)$ and $\bar{\beta}=\left(\beta_{1}, \ldots, \beta_{r}\right)$, with $\alpha_{1}, \ldots, \alpha_{r}, \beta_{1}, \ldots, \beta_{r} \in \overline{\mathbb{Q}}-\{0\}$ and $\bar{\beta}$ admissible, and let $\tau$ be a real number $>1$. Then, for large $x$,

$$
\#\left\{n \in \mathbb{N}: n \leq x, \sum_{\ell=0}^{n} \frac{a_{\ell}}{\ell !} \in A_{\rho, \tau}\right\}=O_{\bar{\alpha}, \bar{\beta}, \tau}(\log x),
$$

where $\rho$ and the $a_{\ell}$ 's are given by (1.9) and (1.11). Here, the constant implied by the $O_{\bar{\alpha}, \bar{\beta}, \tau}-$ symbol is effectively computable.

We conjecture that in each nontrivial instance of our theorem, the number of matchings of partial sums with partial quotients of the corresponding continued fractions is actually finite. To that end, we performed extensive calculations for the cases $r=2, \alpha_{1}=\alpha_{2}=1$, $\beta_{1}=a / b$, and $b_{2}=c / d$, with $1 \leq|a|,|b|,|c|,|d| \leq 50$. In particular, we examined both partial sums and partial quotients up to 10,000 terms. The number of common values that we found ranged from 0 to 3 . In the following table, we list only a few representative findings.

\begin{tabular}{|c|c|}
\hline$\rho$ & Common Values \\
\hline$e^{2 / 19}+e^{-3 / 19}$ & $\left\{2, \frac{112}{57}\right\}$ \\
$e^{-9 / 10}+e^{4 / 5}$ & $\left\{2, \frac{21}{8}\right\}$ \\
$e^{-1 / 30}+e^{-1 / 15}$ & $\left\{2, \frac{19}{10}, \frac{137}{72}\right\}$ \\
$e^{1 / 21}+e^{2 / 21}$ & $\left\{2, \frac{15}{7}, \frac{737}{343}\right\}$ \\
$e^{1 / 45}+e^{2 / 45}$ & $\left\{2, \frac{31}{15}, \frac{335}{162}\right\}$ \\
\hline
\end{tabular}

For the exponential sums in the table above, we conjecture that we have found all the instances where a partial quotient matches a partial sum. We noticed that for $a=-c=1$ and $b=d$, i.e., when we examined $2 \cosh (1 / b)$, we always found two common values for the partial sums and partial quotients. In fact, it is easy to prove that for $\cosh (1 / b)$, we always have an intersection of $\left\{2,2+1 / b^{2}\right\}$ of partial sums and partial quotients. We conjecture that these are the only coalescing values. Further observations led us to the following theorem, which we do not prove here. It is likely that the two common values in Theorem 1.4 below are the only ones.

Theorem 1.4. For any positive integers $a$ and $c$, and any $n$ that is a multiple of $a+c$ and satisfies the inequality

$$
n>\left\{a+c, \frac{4\left(a^{3}+c^{3}\right)}{21 a c}\right\},
$$

both 2 and $2+(a+c) / n$ are common values of partial sums and partial quotients for

$$
\rho=e^{a / n}+e^{c / n}
$$

\section{PROOF OF THEOREM 1.1}

The proof of Theorem 1.1 follows roughly along the same lines as the proofs of the results from [1] that we delineated in the Introduction. 
Let $\left\{a_{n}\right\}$ be a sequence of nonnegative integers satisfying (1.4) and (1.5), and let $G(s)$ be given by (1.6). Fix a positive rational number $r$ and a real number $\delta>0$. Fix a small $\eta>0$, whose precise value will depend on $\delta$ only. For a fixed large integer $N$, let $S_{G, r, \delta, \eta, N}$ denote the set of integers in the interval $[N,(1+\eta) N]$ for which $a_{n} \neq 0$ and

$$
\sum_{\ell=1}^{n} \frac{a_{\ell}}{\ell !} r^{\ell} \in A_{G(r), \delta}
$$

Next, take any nonempty subset $S$ in $S_{G, r, \delta, \eta, N}$ and denote $v=\#(S)$. By the positivity of $r$ and the nonnegativity of $a_{n}, n \geq 1$, it follows that for any integer $n$,

$$
G(r)-\sum_{\ell=1}^{n} \frac{a_{\ell}}{\ell !} r^{n}=\sum_{\ell=n+1}^{\infty} \frac{a_{\ell}}{\ell !} r^{n} \geq \frac{a_{n+1+i(n)}}{(n+1+i(n)) !} r^{n+1+i(n)} .
$$

By (2.1), (1.4), Stirling's formula, and the fact that $a_{n+1+i(n)} \geq 1$, we see that, uniformly for all integers $n \in[N,(1+\eta) N]$,

$$
G(r)-\sum_{\ell=1}^{n} \frac{a_{\ell}}{\ell !} r^{n}>\frac{1}{n^{n(1+o(1))}}
$$

For those $n \in S_{G, r, \delta, \eta, N}$, we then find from (2.2) that

$$
H\left(\sum_{\ell=1}^{n} \frac{a_{\ell}}{\ell !} r^{n}\right)<\left(G(r)-\sum_{\ell=1}^{n} \frac{a_{\ell}}{\ell !} r^{\ell}\right)^{-1 /(1+\delta)}<n^{\frac{1+o(1)}{1+\delta} n}<N^{\frac{(1+\eta)(1+o(1))}{1+\delta} N}<N^{\left(1-\frac{1}{2} \delta\right) N}
$$

for $0<\delta<1, \eta$ sufficiently small in terms of $\delta$, and $N$ sufficiently large.

Next, let $r=a / b, a, b \in \mathbb{Z}, b \geq 1,(a, b)=1$, and for any $n \in S$, let $A_{n}$ be the positive integer for which

$$
\sum_{\ell=1}^{n} \frac{a_{\ell}}{\ell !} r^{\ell}=\frac{A_{n}}{b^{n} n !}
$$

Denote $D_{n}:=\left(A_{n}, b^{n} n !\right)$. Let $n, m \in S$ with $n<m$. Then

$$
\sum_{\ell=1}^{n} \frac{a_{\ell}}{\ell !} r^{\ell}<\sum_{\ell=1}^{m} \frac{a_{\ell}}{\ell !} r^{\ell}
$$

and

$$
A_{m}-b^{m-n} m(m-1) \cdots(n+1) A_{n} \in \mathbb{N} .
$$

Uniformly, for all $n, m$ as above, we can conclude that

$$
\begin{aligned}
\left(D_{n}, D_{m}\right) & \leq\left(A_{n}, A_{m}\right) \leq A_{m}-b^{m-n} m(m-1) \cdots(n+1) A_{n} \\
& =b^{m} m ! \sum_{\ell=n+1}^{m} \frac{a_{\ell} a^{\ell}}{\ell ! b^{\ell}} \leq(m-n) \frac{m !}{n !} H(r)^{m} \max _{n<\ell \leq m} a_{\ell} \\
& =O\left(\frac{m !}{n !} H(r)^{m} m^{o(m)}\right) .
\end{aligned}
$$


If we let $L_{S}$ denote the least common multiple of the $D_{n}$ 's, with $n \in S$, then, by Stirling's formula, we find that

$$
\frac{\prod_{n \in S} D_{n}}{\prod_{\substack{n, m \in S \\ n<m}}\left(D_{n}, D_{m}\right)} \leq L_{S} \leq b^{[(1+\eta) N]}[(1+\eta) N] ! \ll_{\eta}\left(\frac{2 b N}{e}\right)^{(1+\eta) N} .
$$

Now, by the definition of $D_{n}$,

$$
H\left(\sum_{\ell=1}^{n} \frac{a_{\ell}}{\ell !} r^{\ell}\right)=H\left(\frac{A_{n}}{b^{n} n !}\right)=\max \left\{\frac{\left|A_{n}\right|}{D_{n}}, \frac{b^{n} n !}{D_{n}}\right\} \sim \frac{b^{n} n !}{D_{n}} \max \{1,|G(r)|\},
$$

as $n \rightarrow \infty$, and so by (2.7), (2.3), and Stirling's formula,

$$
D_{n} \geq \frac{b^{n} n !}{N^{\left(1-\frac{1}{2} \delta\right) N}} \geq\left(\frac{b}{e}\right)^{N} N^{\delta N / 2}
$$

By (2.5)-(2.8), we find that

$$
\begin{aligned}
\left(\frac{b}{e}\right)^{v N} N^{\delta v N / 2} & \ll_{\eta, v}\left(\frac{2 b N}{e}\right)^{(1+\eta) N} \prod_{\substack{n, m \in S \\
n<m}} \frac{m !}{n !} H(r)^{m} m^{o(m)} \\
& \ll_{\eta, v}\left(\frac{2 b N}{e}\right)^{(1+\eta) N} H(r)^{\frac{1}{2}(1+\eta) N v(v-1)} N^{o\left(v^{2} N\right)} \prod_{\substack{n, m \in S \\
n<m}} \frac{m !}{n !} .
\end{aligned}
$$

Observe that the product on the right side of (2.9) can be written in the simplified form

$$
\prod_{\substack{n, m \in S \\ n<m}} \frac{m !}{n !}=\prod_{n \in S}(n !)^{v-1-2 \#\{m \in S: m>n\}} .
$$

On the right-hand side of (2.10), we use the inequality $n ! \geq N$ ! for those $n \in S$ for which $v-1-2 \#\{m \in S: m>n\}<0$ and the inequality $n ! \leq[(1+\eta) N]$ ! for those $n \in S$ for which $v-1-2 \#\{m \in S: m>n\}>0$. It follows that

$$
\begin{aligned}
\prod_{\substack{n, m \in S \\
n<m}} \frac{m !}{n !} & \leq \begin{cases}\left(\frac{[(1+\eta) N] !}{N !}\right)^{\left(v^{2}-1\right) / 4}, & \text { if } v \text { is odd } \\
\left(\frac{[(1+\eta) N] !}{N !}\right)^{v^{2} / 4}, & \text { if } v \text { is even },\end{cases} \\
& \leq\left(\frac{[(1+\eta) N] !}{N !}\right)^{v^{2} / 4}
\end{aligned}
$$

By (2.9), (2.11), and Stirling's formula, we find that

$$
\begin{aligned}
\frac{\delta v N}{2} \log N \leq & (1+\eta) N \log \frac{2 b N}{e}+\frac{(1+\eta) N v(v-1)}{2} \log H(r) \\
& +\frac{v^{2}}{4}\left((1+\eta) N \log \frac{(1+\eta) N}{e}-N \log \frac{N}{e}\right)+o\left(v^{2} N \log N\right) .
\end{aligned}
$$


We now let $N \rightarrow \infty$ while keeping $\delta, \eta$, and $v$ fixed. It follows that

$$
\frac{\delta v}{2} \leq 1+\eta+\frac{v^{2} \eta}{4}+o\left(v^{2}\right)
$$

We see that the inequality (2.13) fails to hold if $N$ is large enough, because, for a fixed $\delta>0$ and a given positive integer $v$, we can choose $\eta$ small enough so that

$$
\frac{\delta v}{2}>1+\eta+\frac{v^{2} \eta}{4}
$$

which contradicts (2.13) for $N$ sufficiently large.

In conclusion, for such a fixed $v$ and any $N$ sufficiently large, $S_{G, r, \delta, \eta, N}$ cannot have $v$ elements or more. Lastly, we take a sufficiently large integer $N$, subdivide the interval $[1, N]$ into intervals of the form $[T,(1+\eta) T]$, and use the upper bound obtained above for each such interval, provided that $T$ is sufficiently large. We thus readily obtain the bound (1.7) to complete the proof of Theorem 1.1 .

\section{Proof OF TheOREM 1.2}

Let $x$ be large, and let $p_{n}, n \geq 1$, be the $n$-th prime. Define $r$ by

$$
2 \cdot 5 \cdot 7 \cdots p_{r} \leq x<2 \cdot 5 \cdot 7 \cdots p_{r} \cdot p_{r+1} .
$$

Then, by (3.1) and the prime number theorem, we have

$$
\log x \sim \sum_{\substack{p \leq p_{r+1} \\ p \text { prime }}} \log p \sim p_{r+1}
$$

Define

$$
q=2 \prod_{n=3}^{r} p_{n}
$$

By (3.1), we have $q \leq x$. Now, choose $\chi$ to be the principal character modulo $q$. Then,

$$
\chi(n)= \begin{cases}1, & \text { if } n=1,3,3^{2}, 3^{3}, \ldots, \\ 0, & \text { if } n<p_{r+1} \text { and } n \neq 1,3,3^{2}, 3^{3}, \ldots,\end{cases}
$$

and

$$
\alpha:=\sum_{n \geq 1} \frac{\chi(n)}{n !}=1+\frac{1}{3 !}+\frac{1}{3^{2} !}+\frac{1}{3^{3 !}}+\cdots+\sum_{n \geq p_{r+1}} \frac{\chi(n)}{n !} .
$$

We now let $k$ be a nonnegative integer such that $3^{k+1}<p_{r+1}$, and we define $a_{k}$ as follows:

$$
1+\frac{1}{3 !}+\frac{1}{3^{2 !}}+\frac{1}{3^{3 !}}+\cdots+\frac{1}{\left(3^{k}\right) !}=\frac{a_{k}}{3^{k !}} .
$$

Then,

$$
\left|\alpha-\frac{a_{k}}{3^{k} !}\right|<\sum_{n \geq\left(3^{k+1}\right) !} \frac{1}{n !} \leq \frac{2}{\left(3^{k+1}\right) !}<\frac{1}{2\left(3^{k} !\right)^{2}}
$$


Recalling that for a rational number $a / b$, if $|\alpha-a / b|<1 /\left(2 b^{2}\right)$, then $a / b$ is a convergent for the continued fraction of $\alpha$, we can see that $a_{k} /\left(3^{k} !\right)$ is a convergent and a partial sum of $\alpha$. Thus,

$$
\ell(\chi)>>\log p_{r+1} \sim \log \log x,
$$

by (3.2), which completes the proof.

\section{Proof of Theorem 1.3}

The proof of Theorem 1.3 is based on several lemmas.

Lemma 4.1. Let $\bar{\alpha}=\left(\alpha_{1}, \ldots, \alpha_{r}\right)$ and $\bar{\beta}=\left(\beta_{1}, \ldots, \beta_{r}\right)$, with $\alpha_{1}, \ldots, \alpha_{r}, \beta_{1}, \ldots, \beta_{r} \in$ $\overline{\mathbb{Q}}-\{0\}$ and $\bar{\beta}$ admissible. There exists a positive integer $N_{\bar{\alpha}, \bar{\beta}}$, depending only on $\bar{\alpha}$ and $\bar{\beta}$, with the following property. For any integers $m$ and $n$ satisfying

$$
\min \{m, n\} \geq N_{\bar{\alpha}, \bar{\beta}} \text { and }|m-n| \geq r,
$$

the partial sums $\sum_{\ell=0}^{m} \frac{a_{\ell}}{\ell !}$ and $\sum_{\ell=0}^{n} \frac{a_{\ell}}{\ell !}$, with $a_{\ell}$ given by (1.11), are distinct.

Proof of Lemma 4.1. Let $\bar{\alpha}$ and $\bar{\beta}$ be as in the statement of the lemma, and fix a $j_{0} \in$ $\{1, \ldots, r\}$ for which

$$
\left|\beta_{j_{0}}\right|=\max \left\{\left|\beta_{1}\right|,\left|\beta_{2}\right|, \ldots,\left|\beta_{r}\right|\right\}
$$

Let

$$
\mathscr{S}=\left\{j \in\{1, \ldots, r\}:\left|\beta_{j}\right|=\left|\beta_{j_{0}}\right|\right\} .
$$

Since $\bar{\beta}$ is admissible, we know that for any $j \in \mathscr{S}, \beta_{j} / \beta_{j_{0}}$ is a root of unity. Therefore, the function given by

$$
\ell \longmapsto \sum_{j \in \mathscr{S}} \frac{\alpha_{j}}{\alpha_{j_{0}}}\left(\frac{\beta_{j}}{\beta_{j_{0}}}\right)^{\ell}
$$

takes only finitely many values as $\ell$ runs over $\mathbb{N}$. Hence, there exists a constant $\epsilon_{\bar{\alpha}, \bar{\beta}} \geq 0$ depending only on $\bar{\alpha}$ and $\bar{\beta}$, such that for any $\ell \in \mathbb{N}$,

$$
\sum_{j \in \mathscr{S}} \frac{\alpha_{j}}{\alpha_{j_{0}}}\left(\frac{\beta_{j}}{\beta_{j_{0}}}\right)^{\ell}=0 \quad \text { or } \quad\left|\sum_{j \in \mathscr{S}} \frac{\alpha_{j}}{\alpha_{j_{0}}}\left(\frac{\beta_{j}}{\beta_{j_{0}}}\right)^{\ell}\right| \geq \epsilon_{\bar{\alpha}, \bar{\beta}} .
$$

In other words, for any $\ell \in \mathbb{N}$,

$$
\sum_{j \in \mathscr{S}} \alpha_{j} \beta_{j}^{\ell}=0 \quad \text { or } \quad\left|\sum_{j \in \mathscr{S}} \alpha_{j} \beta_{j}^{\ell}\right| \geq \epsilon_{\bar{\alpha}, \bar{\beta}}\left|\alpha_{j_{0}}\right|\left|\beta_{j_{0}}\right|^{\ell} .
$$

In the case $\mathscr{S}$ does not coincide with the entire set $\{1, \ldots, r\}$, fix a $j_{1} \in\{1, \ldots, r\}-\mathscr{S}$ for which

$$
\left|\beta_{j_{1}}\right|=\max \left\{\left|\beta_{j}\right|: j \in\{1, \ldots, r\}-\mathscr{S}\right\} .
$$

Then, for all $\ell$,

$$
\left|\sum_{j \in\{1, \ldots, r\}-\mathscr{S}} \alpha_{j} \beta_{j}^{\ell}\right|=O_{\bar{\alpha}}\left(\left|\beta_{j_{1}}\right|^{\ell}\right)
$$


Since $\left|\beta_{j_{1}}\right|<\left|\beta_{j_{0}}\right|$, the upper bound on the right side of (4.8) is exponentially small compared to the lower bound on the right side of (4.6) as $\ell \rightarrow \infty$. By (1.11), (4.6), and (4.8), for all $\ell$ sufficiently large,

$$
\left|a_{\ell}\right|=O_{\bar{\alpha}}\left(\left|\beta_{j_{1}}\right|^{\ell}\right) \quad \text { or } \quad\left|a_{\ell}\right| \geq \frac{1}{2} \epsilon_{\bar{\alpha}, \bar{\beta}}\left|\alpha_{j_{0}}\right|\left|\beta_{j_{0}}\right|^{\ell} \text {. }
$$

Next, let us remark that the first alternative in (4.6) cannot happen for $\#(\mathscr{S})$ consecutive values of $\ell$. Indeed, assume that, for some $m$,

$$
\sum_{j \in \mathscr{S}} \alpha_{j} \beta_{j}^{\ell}=0, \quad \text { for } \quad \ell=m+1, m+2, \ldots, m+\#(\mathscr{S}) .
$$

The associated Vandermonde determinant is nonzero, since the $\beta_{j}$ 's are distinct; hence the system (4.10) can only have the trivial solution $\bar{\alpha}=\overline{0}$, which is not the case here. Therefore, since $\#(\mathscr{S}) \leq r$, in any set of $r$ consecutive values of $\ell$, at least one of them satisfies the second alternative in (4.6). It follows that the second alternative in (4.9) holds at least once in any $r$ consecutive large values of $\ell$.

Now let $m$ and $n$ be large positive integers with $n \geq m+r$. Let $\ell_{0}$ be the smallest value of $\ell$ in $\{m+1, \ldots, n\}$ for which the second alternative in (4.9) holds. Thus, $\ell_{0} \leq m+r$. It follows that

$$
\left|a_{\ell}\right|=O_{\bar{\alpha}, \bar{\beta}}\left(\left|\beta_{j_{1}}\right|^{\ell}\right), \quad \text { for } \quad m+1 \leq \ell<\ell_{0},
$$

and

$$
\left|a_{\ell_{0}}\right| \geq \frac{1}{2} \epsilon_{\bar{\alpha}, \bar{\beta}}\left|\alpha_{j_{0}}\right|\left|\beta_{j_{0}}\right|^{\ell_{0}}
$$

Also, trivially from (1.11),

$$
\left|a_{\ell}\right|=O_{\bar{\alpha}}\left(\left|\beta_{j_{0}}\right|^{\ell}\right), \quad \text { for all } \quad \ell>\ell_{0}
$$

By (4.13) and (4.12),

$$
\sum_{\ell_{0}<\ell \leq n} \frac{\left|a_{\ell}\right|}{\ell !}=O_{\bar{\alpha}}\left(\sum_{\ell=\ell_{0}+1}^{n} \frac{\left|\beta_{j_{0}}\right|^{\ell}}{\ell !}\right)=O_{\bar{\alpha}, \bar{\beta}}\left(\frac{\left|\beta_{j_{0}}\right|^{\ell}}{m\left(\ell_{0}\right) !}\right)=O_{\bar{\alpha}, \bar{\beta}}\left(\frac{\left|a_{\ell_{0}}\right|}{m\left(\ell_{0}\right) !}\right) .
$$

Also, by (4.11) and (4.12),

$$
\sum_{m<\ell<\ell_{0}} \frac{\left|a_{\ell}\right|}{\ell !}=O_{\bar{\alpha}, \bar{\beta}}\left(\frac{\left|\beta_{j_{1}}\right|^{\ell_{0}}}{(m+1) !}\right)=O_{\bar{\alpha}, \bar{\beta}}\left(m^{\ell_{0}-m}\left(\frac{\beta_{j_{1}}}{\beta_{j_{0}}}\right)^{m} \frac{\left|a_{\ell_{0}}\right|}{\left(\ell_{0}\right) !}\right) .
$$

For $m$ large, the far right-hand sides of (4.14) and (4.15) are negligible compared to $\left|a_{\ell_{0}}\right| /\left(\ell_{0}\right)$ !; therefore

$$
\begin{aligned}
\left|\sum_{\ell=0}^{n} \frac{a_{\ell}}{\ell !}-\sum_{\ell=0}^{m} \frac{a_{\ell}}{\ell !}\right|=\left|\sum_{\ell=m+1}^{n} \frac{a_{\ell}}{\ell !}\right| & \geq \frac{a_{\ell_{0}}}{\left(\ell_{0}\right) !}-\sum_{\substack{m<\ell<n \\
\ell \neq \ell_{0}}} \frac{a_{\ell}}{\ell !} \\
& \geq \frac{a_{\ell_{0}}}{2\left(\ell_{0}\right) !} \geq \frac{\epsilon_{\bar{\alpha}, \bar{\beta}}\left|\alpha_{j_{0}}\right|\left|\beta_{j_{0}}\right|^{\ell_{0}}}{4\left(\ell_{0}\right) !} .
\end{aligned}
$$

This shows that the partial sums $\sum_{\ell=0}^{n} \frac{a_{\ell}}{\ell !}$ and $\sum_{\ell=0}^{m} \frac{a_{\ell}}{\ell !}$ are distinct, and completes the proof of the lemma. 
Lemma 4.2. Let $\bar{\alpha}=\left(\alpha_{1}, \ldots, \alpha_{r}\right)$ and $\bar{\beta}=\left(\beta_{1}, \ldots, \beta_{r}\right)$, with $\alpha_{1}, \ldots, \alpha_{r}, \beta_{1}, \ldots, \beta_{r} \in$ $\overline{\mathbb{Q}}-\{0\}$ and $\bar{\beta}$ admissible, and let $\rho$ be given by (1.9). There exist a positive integer $N_{\bar{\alpha}, \bar{\beta}}^{\prime}$ and an $\epsilon_{\bar{\alpha}, \bar{\beta}}^{\prime}>0$, depending only on $\bar{\alpha}$ and $\bar{\beta}$, such that for all $m \geq N_{\bar{\alpha}, \bar{\beta}}^{\prime}$,

$$
\left|\rho-\sum_{\ell=0}^{m} \frac{a_{\ell}}{\ell !}\right| \geq \frac{\epsilon_{\bar{\alpha}, \bar{\beta}}^{\prime}\left(\max \left\{\left|\beta_{1}\right|, \ldots,\left|\beta_{r}\right|\right\}\right)^{m}}{m^{r} m !} .
$$

Proof of Lemma 4.2. The computations from the proof of Lemma 4.1 presented above were detailed enough to immediately imply Lemma 4.2 as well. More precisely, using (4.16) in combination with the fact that $\ell_{0} \leq m+r$, we find that

$$
\left|\sum_{\ell=0}^{n} \frac{a_{\ell}}{\ell !}-\sum_{\ell=0}^{m} \frac{a_{\ell}}{\ell !}\right| \geq \frac{\epsilon_{\bar{\alpha}, \bar{\beta}}^{\prime}\left|\beta_{j_{0}}\right|^{m}}{m^{r} m !},
$$

for some constant $\epsilon_{\bar{\alpha}, \bar{\beta}}^{\prime}>0$ depending only on $\bar{\alpha}$ and $\bar{\beta}$, any $m$ large enough, and any $n \geq m+r$. The right side of (4.18) is independent of $n$. Letting $n \rightarrow \infty$ while keeping $m$ fixed, and recalling the definition of $\left|\beta_{j_{0}}\right|$, we find that (4.17) holds for all $m$ large enough. This completes the proof of Lemma 4.2.

Next, let $\bar{\alpha}=\left(\alpha_{1}, \ldots, \alpha_{r}\right)$ and $\bar{\beta}=\left(\beta_{1}, \ldots, \beta_{r}\right)$, with $\alpha_{1}, \ldots, \alpha_{r}, \beta_{1}, \ldots, \beta_{r} \in \overline{\mathbb{Q}}-\{0\}$ and $\bar{\beta}$ admissible, and consider the number field

$$
K:=\mathbb{Q}\left(\alpha_{1}, \ldots, \alpha_{r}, \beta_{1}, \ldots, \beta_{r}\right) .
$$

Denote

$$
b:=\min \left\{d \in \mathbb{Z}: d \geq 1, d \alpha_{1}, \ldots, d \alpha_{r}, d \beta_{1}, \ldots, d \beta_{r} \in O_{K}\right\} .
$$

With the $a_{\ell}$ 's defined by (1.11), $b^{\ell+1} a_{\ell}$ belongs to $O_{K}$, for each $\rho$. Therefore, writing each partial sum of the series from the right side of (1.10) in the form

$$
\sum_{\ell=0}^{n} \frac{a_{\ell}}{\ell !}=\frac{A_{n}}{b^{n+1} n !}
$$

we have $A_{n} \in O_{K}$ for all $n$. We consider the ideal of $O_{K}$ generated by $A_{n}$ and $b^{n+1} n$ !,

$$
J_{n}:=\left(A_{n}, b^{n+1} n !\right), \quad n=0,1,2, \ldots,
$$

and examine the size of the norm $\left\|J_{n}\right\|$ of $J_{n}$. One would naturally expect to have very little cancellation on the right side of (4.21), and $\left\|J_{n}\right\|$ to be relatively small. Notice that if for some $n$ the partial sum $\sum_{\ell=0}^{n} \frac{a_{\ell}}{\ell !}$ is zero, then $A_{n}=0$, and the norm of $J_{n}$ is as large as it can be, $\left\|J_{n}\right\|=\left(b^{n+1} n !\right)^{[K: \mathbb{Q}]}$. This only happens finitely many times, since $\sum_{\ell=0}^{n} \frac{a_{\ell}}{\ell !} \rightarrow \rho$ as $n \rightarrow \infty$, and $\rho \neq 0$. Notice also that if $\left\|J_{n}\right\|$ is unusually large for one value of $n$, it will automatically be large for other nearby values of $n$ that happen to produce the exact same partial sum. By Lemma 4.1, this phenomenon is harmless, since the multiplicity of each partial sum $\sum_{\ell=0}^{n} \frac{a_{\ell}}{\ell !}$, with $n$ sufficiently large, is bounded by $r$. The following lemma, which generalizes to number fields a key result from [1], shows that with at most a logarithmic number of exceptions, $\left\|J_{n}\right\|$ is bounded by $n^{\epsilon n}$, for any fixed $\epsilon>0$, and $n$ sufficiently large. 
Lemma 4.3. Let $\bar{\alpha}=\left(\alpha_{1}, \ldots, \alpha_{r}\right)$ and $\bar{\beta}=\left(\beta_{1}, \ldots, \beta_{r}\right)$, with $\alpha_{1}, \ldots, \alpha_{r}, \beta_{1}, \ldots, \beta_{r} \in$ $\overline{\mathbb{Q}}-\{0\}$ and $\bar{\beta}$ admissible. For any $\epsilon>0$, there exists an effectively computable constant $C_{\bar{\alpha}, \bar{\beta}, \epsilon}^{\prime}>0$ such that for all sufficiently large real numbers $x$,

$$
\#\left\{n \in \mathbb{Z}: 1 \leq n \leq x,\left\|J_{n}\right\| \geq n^{\epsilon n}\right\} \leq C_{\bar{\alpha}, \bar{\beta}, \epsilon}^{\prime} \log x,
$$

where $J_{n}$ is defined via (1.11) and (4.19)-(4.22).

Proof of Lemma 4.3. Let $\bar{\alpha}$ and $\bar{\beta}$ be as in the statement of the lemma and fix an $\epsilon>0$. We choose a small $\eta>0$, whose precise value will be given later, and will be allowed to depend on $\epsilon$ and on the degree of $K=\mathbb{Q}\left(\alpha_{1}, \ldots, \alpha_{r}, \beta_{1}, \ldots, \beta_{r}\right)$ over $\mathbb{Q}$. It suffices to show that there exists an effectively computable constant $C_{\bar{\alpha}, \bar{\beta}, \epsilon, \eta}^{\prime \prime}$ and a positive integer $N_{\bar{\alpha}, \bar{\beta}, \epsilon, \eta}^{\prime \prime}$, such that for any integer $N \geq N_{\bar{\alpha}, \bar{\beta}, \epsilon, \eta}^{\prime \prime}$,

$$
\#\left\{n \in \mathbb{Z}: N \leq n \leq N(1+\eta),\left\|J_{n}\right\| \geq n^{\epsilon n}\right\} \leq C_{\bar{\alpha}, \bar{\beta}, \epsilon, \eta}^{\prime \prime} .
$$

Then (4.23) follows immediately from (4.24), by writing the interval $\left[N_{\bar{\alpha}, \bar{\beta}, \epsilon, \eta}^{\prime \prime}, x\right]$ as a union of at most $\left\lceil\frac{\log x-\log N_{\bar{\alpha}, \bar{\beta}, \epsilon, \eta}^{\prime \prime}}{\log (1+\eta)}\right\rceil$ intervals of the form $[N, N(1+\eta)]$. In order to prove (4.24), consider such an interval $[N, N(1+\eta)]$ with $N$ large. By requiring $N_{\bar{\alpha}, \bar{\beta}, \epsilon, \eta}^{\prime \prime}$ to be larger than the positive integer $N_{\bar{\alpha}, \bar{\beta}}$ from the statement of Lemma 4.1, we may assume in what follows that $N \geq N_{\bar{\alpha}, \bar{\beta}}$. Consider the exceptional set

$$
\mathcal{E}_{\bar{\alpha}, \bar{\beta}, \epsilon, \eta, N}:=\left\{n \in \mathbb{Z}: N \leq n \leq N(1+\eta),\left\|J_{n}\right\| \geq n^{\epsilon n}\right\},
$$

and construct a subset $\mathcal{E}_{\bar{\alpha}, \bar{\beta}, \epsilon, \eta, N}^{*}$ of $\mathcal{E}_{\bar{\alpha}, \bar{\beta}, \epsilon, \eta, N}$ as follows. Let $n_{1}$ be the smallest element of $\mathcal{E}_{\bar{\alpha}, \bar{\beta}, \epsilon, \eta, N}$. Let $n_{2}$ be the smallest element of $\mathcal{E}_{\bar{\alpha}, \bar{\beta}, \epsilon, \eta, N}$ that is $\geq n_{1}+r$, let $n_{3}$ be the smallest element of $\mathcal{E}_{\bar{\alpha}, \bar{\beta}, \epsilon, \eta, N}$ that is $\geq n_{2}+r$, and so on, until this procedure stops. Then set

$$
\mathcal{E}_{\bar{\alpha}, \bar{\beta}, \epsilon, \eta, N}^{*}=\left\{n_{1}, n_{2}, n_{3}, \ldots\right\} .
$$

By the foregoing construction, $\#\left(\mathcal{E}_{\bar{\alpha}, \bar{\beta}, \epsilon, \eta, N}\right) \leq r \#\left(\mathcal{E}_{\bar{\alpha}, \bar{\beta}, \epsilon, \eta, N}^{*}\right)$, and so (4.24) will be proved if we show that there is an effectively computable constant $C_{\bar{\alpha}, \bar{\beta}, \epsilon, \eta}^{\prime \prime \prime}$ such that, uniformly for all $N$ sufficiently large,

$$
\#\left(\mathcal{E}_{\bar{\alpha}, \bar{\beta}, \epsilon, \eta, N}^{*}\right) \leq C_{\bar{\alpha}, \bar{\beta}, \epsilon, \eta}^{\prime \prime \prime}
$$

Fix now a positive integer $k$ satisfying

$$
k \leq \#\left(\mathcal{E}_{\bar{\alpha}, \bar{\beta}, \epsilon, \eta, N}^{*}\right) .
$$

As a side remark, we emphasize that the argument below would not work if instead of (4.28) we let $k$ equal $\#\left(\mathcal{E}_{\bar{\alpha}, \bar{\beta}, \epsilon, \eta, N}^{*}\right)$ directly. The reason is that in the argument below $k$ needs to be in a suitable range; if $k$ is too small or too large, we do not obtain a contradiction.

To proceed, let $J$ denote the least common multiple of the ideals $J_{n_{1}}, J_{n_{2}}, \ldots, J_{n_{k}}$. For $1 \leq i<j \leq k$, let $J_{i j}$ denote the greatest common divisor of the ideals $J_{n_{i}}$ and $J_{n_{j}}$. Let $\mathcal{P}$ be an arbitrary prime ideal of $O_{K}$, and let $e_{1}, e_{2}, \ldots, e_{k}$ denote the exponents of $\mathcal{P}$ in $J_{n_{1}}, J_{n_{2}}, \ldots, J_{n_{k}}$, respectively. Then the exponent of $\mathcal{P}$ in $J$ equals $\max \left\{e_{1}, e_{2}, \ldots, e_{k}\right\}$, the exponent of $P$ in the product $\prod_{1 \leq j \leq k} J_{n_{j}}$ equals the sum $\sum_{1 \leq j \leq k} e_{j}$, and for any $1 \leq$ 
$i<j \leq k$, the exponent of $\mathcal{P}$ in $J_{i j}$ equals $\min \left\{e_{i}, e_{j}\right\}$. Note that the nonnegative integers $e_{1}, e_{2}, \ldots, e_{k}$ always satisfy the inequality

$$
e_{1}+e_{2}+\cdots+e_{k} \leq \max \left\{e_{1}, e_{2}, \ldots, e_{k}\right\}+\sum_{1 \leq i<j \leq k} \min \left\{e_{i}, e_{j}\right\} .
$$

Indeed, let $s \in\{1,2, \ldots, k\}$ be such that $e_{s}=\max \left\{e_{1}, e_{2}, \ldots, e_{k}\right\}$. Then $e_{s}$ appears as the first term on the right side of (4.29), each term $e_{i}$ on the left side with $i<s$ also appears on the right side as $\min \left\{e_{i}, e_{s}\right\}$, and each term $e_{j}$ on the left side with $j>s$ also appears on the right side as $\min \left\{e_{s}, e_{j}\right\}$. Also, all the remaining terms on the right side are nonnegative.

Since the previous discussion applies to all prime ideals of $O_{K}$, we see that

$$
\prod_{1 \leq j \leq k} J_{n_{j}} \text { divides } J \prod_{1 \leq i<j \leq k} J_{i j}
$$

Since $n_{1}, \ldots, n_{k}$ belong to the exceptional set $\mathcal{E}_{\bar{\alpha}, \bar{\beta}, \epsilon, \eta, N}$,

$$
\prod_{1 \leq j \leq k}\left\|J_{n_{j}}\right\| \geq \prod_{1 \leq j \leq k} n_{j}^{\epsilon n_{j}} \geq N^{k \epsilon N}
$$

On the other hand, for each $j, J_{n_{j}}$ divides the principal ideal $\left(b^{n_{j}+1} n_{j} !\right)$, which in turn divides $\left(b^{\lfloor N(1+\eta)+1\rfloor}\lfloor N(1+\eta)\rfloor !\right)$, and therefore $J$ also divides this ideal. Taking norms, we find that

$$
\|J\| \leq b^{[K: \mathbb{Q}]\lfloor N(1+\eta)+1\rfloor}(\lfloor N(1+\eta)\rfloor !)^{[K: \mathbb{Q}]} .
$$

Combining (4.32) with Stirling's formula, we see that for $N$ sufficiently large,

$$
\|J\| \leq N^{N(1+2 \eta)[K: \mathbb{Q}]} .
$$

By (4.30), (4.31), and (4.33), for $N$ sufficiently large,

$$
N^{k \epsilon N} \leq N^{N(1+2 \eta)[K: \mathbb{Q}]} \prod_{1 \leq i<j \leq k}\left\|J_{i j}\right\| .
$$

Lastly, in order to bound the product on the right side of (4.34), recall that by Lemma 4.1, the partial sums $\sum_{\ell=0}^{n_{j}} \frac{a_{\ell}}{\ell !}$, with $1 \leq j \leq k$, are distinct. It follows that for each pair $(i, j)$, $1 \leq i<j \leq k$, the number $w_{i, j}$ defined by

$$
w_{i, j}:=b^{n_{j}+1} n_{j} !\left(\sum_{\ell=0}^{n_{j}} \frac{a_{\ell}}{\ell !}-\sum_{\ell=0}^{n_{i}} \frac{a_{\ell}}{\ell !}\right)=A_{n_{j}}-\frac{b^{n_{j}-n_{i}} n_{j} ! A_{n_{i}}}{n_{i} !}
$$

is a nonzero algebraic integer. Moreover, since $A_{n_{j}} \in J_{n_{j}} \subseteq J_{i j}$ and $A_{n_{i}} \in J_{n_{i}} \subseteq J_{i j}$, by (4.35), we see that $w_{i, j}$ belongs to $J_{i j}$. Therefore,

$$
\| J_{i j}|| \leq\left|N_{K / \mathbb{Q}}\left(w_{i, j}\right)\right|, \quad \text { for } \quad 1 \leq i<j \leq k .
$$

Next, we need an alternative way to bound the norm of each element $w_{i, j}$. We start by rewriting (4.35) as

$$
\begin{aligned}
w_{i, j} & =b^{n_{j}+1} n_{j} ! \sum_{n_{i}<\ell \leq n_{j}} \frac{a_{\ell}}{\ell !} \\
& =b^{n_{j}+1}\left(a_{n_{j}}+n_{j} a_{n_{j}-1}+\cdots+n_{j}\left(n_{j}-1\right) \cdots\left(n_{i}+1\right) a_{n_{i}}\right) .
\end{aligned}
$$


For any embedding $\sigma$ of $K$ into $\mathbb{C}$,

$$
\sigma\left(w_{i, j}\right)=b^{n_{j}+1}\left(\sigma\left(a_{n_{j}}\right)+n_{j} \sigma\left(a_{n_{j}-1}\right)+\cdots+n_{j}\left(n_{j}-1\right) \cdots\left(n_{i}+1\right) \sigma\left(a_{n_{i}}\right)\right) .
$$

By (1.11), for each $\sigma$ and each $\ell$,

$$
\sigma\left(a_{\ell}\right)=\sum_{j=1}^{r} \sigma\left(\alpha_{j}\right) \sigma\left(\beta_{j}\right)^{\ell} .
$$

If we denote

$$
\|\bar{\alpha}\|=\max _{\sigma} \max _{1 \leq j \leq r}\left|\sigma\left(\alpha_{j}\right)\right|
$$

and similarly

$$
\|\bar{\beta}\|=\max _{\sigma} \max _{1 \leq j \leq r}\left|\sigma\left(\beta_{j}\right)\right|
$$

then by (4.39)-(4.41),

$$
\left|\sigma\left(a_{\ell}\right)\right| \leq r\left\|\overline { \alpha } \left|\|\mid \bar{\beta}\|^{\ell}\right.\right.
$$

for any $\sigma$ and $\ell$. Combining (4.38) and (4.42), we find that

$$
\left|\sigma\left(w_{i, j}\right)\right| \leq r\|\bar{\alpha}\| b^{n_{j}+1} \max \left\{\|\bar{\beta}\|^{n_{i}},\|\bar{\beta}\|^{n_{j}}\right\} n_{j}^{n_{j}-n_{i}+1} .
$$

Since $n_{j} \leq N(1+\eta)$ and $n_{j}-n_{i} \leq \eta N$, we see that for $N$ sufficiently large,

$$
\left|\sigma\left(w_{i, j}\right)\right| \leq N^{2 \eta N}
$$

uniformly for $1 \leq i<j \leq k$ and all embeddings $\sigma$ of $K$ into $\mathbb{C}$. Therefore,

$$
\left|N_{K / \mathbb{Q}}\left(w_{i, j}\right)\right|=\prod_{\sigma}\left|\sigma\left(w_{i, j}\right)\right| \leq N^{2 \eta N[K: \mathbb{Q}]},
$$

for $1 \leq i<j \leq k$. Combining (4.34), (4.36), and (4.45), we deduce that

$$
k \epsilon \leq(1+2 \eta)[K: \mathbb{Q}]+k(k-1) \eta[K: \mathbb{Q}] .
$$

With $\epsilon$ and the degree $[K: \mathbb{Q}]$ fixed, if $\eta$ is chosen small enough, then the function given by

$$
t \longmapsto(1+2 \eta)[K: \mathbb{Q}]+t(t-1) \eta[K: \mathbb{Q}]-t \epsilon
$$

has two real and positive roots $t_{1}<t_{2}$. Moreover, denoting $d=[K: \mathbb{Q}]$,

$$
t_{2}-t_{1}=\frac{\sqrt{(\epsilon+\eta d)^{2}-4 \eta(1+2 \eta) d^{2}}}{\eta d} .
$$

For fixed $d$ and $\epsilon$, the right-hand side of (4.48) tends to infinity as $\eta \rightarrow 0$. One can choose an effectively computable $\eta=\eta_{d, \epsilon}>0$, depending only on $d$ and $\epsilon$, such that $\left(\epsilon+\eta_{d, \epsilon} d\right)^{2}-$ $4 \eta_{d, \epsilon}\left(1+2 \eta_{d, \epsilon}\right) d^{2}>0$ and such that the right-hand side of (4.48) is larger than 1 . One can then choose an effectively computable positive integer $k=k_{d, \epsilon}$, depending only on $d$ and $\epsilon$, such that $t_{1}<k_{d, \epsilon}<t_{2}$.

In conclusion, since (4.46) fails for $\eta_{d, \epsilon}$ and $k_{d, \epsilon}$, it follows that (4.28) fails for $\eta_{d, \epsilon}$ and $k_{d, \epsilon}$, for every single sufficiently large $N$. In other words, (4.27) holds for all $N$ sufficiently large, with $\eta=\eta_{d, \epsilon}$ and $C_{\bar{\alpha}, \bar{\beta}, \epsilon, \eta_{d, \epsilon}}^{\prime \prime \prime}=k_{d, \epsilon}-1$. This completes the proof of the lemma. 
Lemma 4.4. Let $\bar{\alpha}=\left(\alpha_{1}, \ldots, \alpha_{r}\right)$ and $\bar{\beta}=\left(\beta_{1}, \ldots, \beta_{r}\right)$, with $\alpha_{1}, \ldots, \alpha_{r}, \beta_{1}, \ldots, \beta_{r} \in$ $\overline{\mathbb{Q}}-\{0\}$ and $\bar{\beta}$ admissible. For any $\epsilon>0$, there exists an effectively computable constant $C_{\bar{\alpha}, \bar{\beta}, \epsilon}>0$, such that for all large $x$,

$$
\#\left\{n \in \mathbb{Z}: 1 \leq n \leq x, H\left(\sum_{\ell=0}^{n} \frac{a_{\ell}}{\ell !}\right) \leq n^{(1-\epsilon) n}\right\} \leq C_{\bar{\alpha}, \bar{\beta}, \epsilon} \log x
$$

where the $a_{\ell}$ 's are given by (1.11).

Proof of Lemma 4.4. Let $\bar{\alpha}$ and $\bar{\beta}$ be as in the statement of the lemma. Fix an $\epsilon>0$ and take a large $x$. By Lemma 4.3, for all $1 \leq n \leq x$ with at most $C_{\bar{\alpha}, \bar{\beta}, \epsilon}^{\prime} \log x$ exceptions, we have

$$
\left\|J_{n}\right\| \leq n^{\epsilon n}
$$

Take such an $n$, and let $d_{n}$ denote the degree of $\sum_{\ell=0}^{n} \frac{a_{\ell}}{\ell !}$ over $\mathbb{Q}$. Let

$$
P_{n}(X)=c_{n, 0} X^{d_{n}}+c_{n, 1} X^{d_{n}-1}+\cdots+c_{n, d_{n}} \in \mathbb{Z}[X]
$$

be irreducible over $\mathbb{Q}$, such that $c_{n, 0}>0, \operatorname{gcd}\left(c_{n, 0}, c_{n, 1}, \ldots, c_{n, d_{n}}\right)=1$, and

$$
P_{n}\left(\sum_{\ell=0}^{n} \frac{a_{\ell}}{\ell !}\right)=0 \text {. }
$$

By (4.21) and (4.51), we derive

$$
c_{n, 0} A_{n}^{d_{n}}+c_{n, 1} A_{n}^{d_{n}-1} b^{n+1} n !+\cdots+c_{n, d_{n}} b^{(n+1) d_{n}}(n !)^{d_{n}}=0 .
$$

It follows from (4.52) that $c_{n, 0} A_{n}^{d_{n}}$ is a multiple of $b^{n+1} n$ ! in $O_{K}$. Let $D_{n}$ denote the greatest common divisor of the positive integers $c_{n, 0}$ and $b^{n+1} n$ !, and write

$$
c_{n, 0}=D_{n} \mu_{n}, \quad b^{n+1} n !=D_{n} \nu_{n}, \quad\left(\mu_{n}, \nu_{n}\right)=1
$$

Then $\mu_{n} A_{n}^{d_{n}}$ is a multiple of $\nu_{n}$ in $O_{K}$, and since $\left(\mu_{n}, \nu_{n}\right)=1$ and $\nu_{n}$ divides $b^{n+1} n$ !, it follows that the principal ideal $\left(\nu_{n}\right)$ divides the ideal generated by $A_{n}^{d_{n}}$ and $b^{n+1} n$ !. Since this ideal divides $J_{n}^{d_{n}}$, it follows that $\left(\nu_{n}\right)$ divides $J_{n}^{d_{n}}$. Taking norms and using (4.50), we find that

$$
\nu_{n}^{[K: \mathbb{Q}]} \leq\left\|J_{n}^{d_{n}}\right\| \leq n^{\epsilon n d_{n}} .
$$

The degree $d_{n}$ is bounded by $[K: \mathbb{Q}]$, and so (4.54) implies that

$$
\nu_{n} \leq n^{\epsilon n}
$$

By (4.53) and (4.55), and the definition of height given by (1.8), we derive that

$$
H\left(\sum_{\ell=0}^{n} \frac{a_{\ell}}{\ell !}\right) \geq c_{n, 0} \geq D_{n}=\frac{b^{n+1} n !}{\nu_{n}} \geq \frac{b^{n+1} n !}{n^{\epsilon n}} .
$$

This completes the proof of the lemma, by an appeal to Stirling's formula. 
Proof of Theorem 1.3. Let $\bar{\alpha}, \bar{\beta}$, and $\tau$ be as in the statement of the theorem. Take a large $x$, and let $n \leq x$ be such that $\sum_{\ell=0}^{n} \frac{a_{\ell}}{\ell !} \in A_{\rho, \tau}$, with $\rho$ given by (1.10). Thus,

$$
\left|\rho-\sum_{\ell=0}^{n} \frac{a_{\ell}}{\ell !}\right| \leq \frac{1}{H\left(\sum_{\ell=0}^{n} \frac{a_{\ell}}{\ell !}\right)^{\tau}} .
$$

Combining (4.57) with Lemma 4.2, for $n \geq N_{\bar{\alpha}, \bar{\beta}}^{\prime}$, we have

$$
H\left(\sum_{\ell=0}^{n} \frac{a_{\ell}}{\ell !}\right) \leq \frac{n^{r / \tau}(n !)^{1 / \tau}}{\left(\epsilon_{\bar{\alpha}, \bar{\beta}}^{\prime}\right)^{1 / \tau}\left(\max \left\{\left|\beta_{1}\right|, \ldots,\left|\beta_{r}\right|\right\}\right)^{n / \tau}} .
$$

Letting now $\epsilon=(1-1 / \tau) / 2$, we see that the right-hand side of $(4.58)$ is $\leq n^{(1-\epsilon) n}$ for $n$ large enough. Thus, Lemma 4.4 is applicable, and in combination with (4.58) completes the proof of the theorem.

\section{REFERENCES}

[1] B. C. Berndt, S. Kim, and A. Zaharescu, Dirichlet L-functions, elliptic curves, hypergeometric functions, and rational approximation with partial sums of power series, Math. Res. Letters, to appear.

[2] B. C. Berndt, S. Kim, and A. Zaharescu, Diophantine approximation of the exponential function and Sondow's Conjecture, Adv. Math., to appear.

[3] H. Davenport, Multiplicative Number Theory, 2nd ed., Springer-Verlag, New York, 1980.

[4] C. S. Davis, Rational approximation to e, J. Austral. Math. Soc. 25 (1978), 497-502.

[5] S. Ramanujan, The Lost Notebook and Other Unpublished Papers, Narosa, New Delhi, 1988.

[6] J. Sondow, A geometric proof that e is irrational and a new measure of its irrationality, Amer. Math. Monthly 113 (2006), 637-641.

[7] J. Sondow and K. Schalm, Which partial sums of the Taylor series for e are convergents to e? (and a link to the primes 2, 5 13, 37, 463). Part II. in Gems in Experimental Mathematics, T. Amdeberhan, L.A. Medina, V.H. Moll, eds., Contemp. Math., vol. 517, American Mathematical Society, Providence, RI, 2010, pp. 349-363.

Department of Mathematics, University of Illinois, 1409 West Green Street, Urbana, IL 61801, USA

E-mail address: berndteillinois.edu

Department of Mathematics, Ohio State University, 231 West 18th Avenue, Columbus, OH 43210, USA

E-mail address: kim.1674@math.ohio-state.edu

Department of Mathematics, University of Illinois, 1409 West Green Street, Urbana, IL 61801, USA

E-mail address: phaovibleillinois.edu

Department of Mathematics, University of Illinois, 1409 West Green Street, Urbana, IL 61801, USA, AND Institute of Mathematics of THE Romanian ACAdEMy, P.O. Box 1-764, BUCHAREST RO-70700, ROMANIA

E-mail address: zaharesc@illinois.edu 